\title{
Case management for frequent emergency department users: no longer a question of if but when, where and how
}

\author{
Mary Malebranche ${ }^{1,2} \cdot$ Véronique S. Grazioli ${ }^{2} \cdot$ Miriam Kasztura $^{2} \cdot$ Catherine Hudon $^{3,4} \cdot$ Patrick Bodenmann $^{2}$
}

Received: 4 June 2020 / Accepted: 6 August 2020 / Published online: 10 December 2020

(c) Canadian Association of Emergency Physicians (CAEP)/ Association Canadienne de Médecine d'Urgence (ACMU) 2020

Keywords Case management $\cdot$ Frequent healthcare use $\cdot$ Implementation $\cdot$ Health services research $\cdot$ Evidence-based medicine

Ten years ago, an editorial entitled "Frequent Users of Emergency Department Services: Gaps in Knowledge and a Proposed Research Agenda" was written by Pines and colleagues describing the evidence surrounding frequent emergency department (ED) use at the time, while proposing ways to advance the field in the years ahead [1]. Citing a handful of studies, Pines et al. concluded that ED-based interventions aimed at reducing frequent ED use showed promise but voiced concern about the generalizability and feasibility of these interventions, highlighting a need for further research. Ten years on, the field of frequent ED use, generally classified as more than 4 ED visits in a year [2], has evolved considerably. Specifically, the evidence supporting ED-based, as well as primary care-based, interventions aimed at frequent users of the ED has grown dramatically thus presenting a unique opportunity to reflect on what we know now and where the field is heading in 2020 and beyond.

Since 2011, at least eight reviews have been published that summarize the evolving evidence supporting interventions aimed at reducing ED use amongst frequent users [3-10]. What is notable is the emergence of case management as the

Mary Malebranche

Mary.malebranche@ucalgary.ca

1 Department of Medicine, Cumming School of Medicine, University of Calgary, HSC 1410, 3330 Hospital Drive, Calgary, AB T2N 4N1, Canada

2 Department of Vulnerabilities and Social Medicine, Center for General Medicine and Public Health (Unisanté), University of Lausanne, Canton of Vaud, Lausanne, Switzerland

3 Department of Family Medicine and Emergency Medicine, Université de Sherbrooke, Sherbrooke, QC, Canada

4 Centre de Recherche du CHUS, Sherbrooke, QC, Canada most widely discussed primary care and ED-based intervention. Other interventions described include individualized care plans, diversion strategies to non-urgent care, social work home visits, and models of patient navigation, but none have garnered the same attention as case management over the years $[5,10,11]$.

Case management, defined as "a collaborative approach to ensure, coordinate and integrate care and services for patients, in which a case manager evaluates, plans, implements, coordinates, and prioritizes services on the basis of patient's needs"[11], has gained global recognition as an intervention that can reduce ED visits and related healthcare costs amongst frequent users [3, 6, 11]. Reductions in ED costs of $26-45 \%$ and inpatient costs of up to $67 \%$ have been cited $[3,4,6]$. When costs of implementing the case management intervention are considered, total hospital expenditures appear similar to usual care but with improved clinical and social outcomes $[3,4,6]$. Other cited benefits include improved patient quality of life and patient satisfaction while also helping achieve more equitable healthcare by reducing barriers to care for frequent ED users who tend to have higher rates of psychiatric and medical comorbidities, higher rates of social isolation, and less financial resources $[1,11]$. By employing a case manager to coordinate and synchronize care across diverse care settings and amongst multiple care providers, addressing both the medical and social needs of these patients, case management interventions can fill gaps in care which no individual care setting (whether ED or primary care) could accomplish alone.

What is more, the emergence of case management as the most widely supported intervention for frequent users of the ED appears largely consistent despite differences in study inclusion criterion across published reviews. Whether focused on general adult frequent users, adults with chronic diseases, or individuals facing homelessness or other 
unstable social conditions, in the vast majority of studies, case management interventions appear to have wide reaching positive impacts.

As a result of this increasingly strong evidence base, questions in the field have begun to shift from whether or not case management is effective to which aspects of case management interventions contribute to positive outcomes? What is the best approach to implementing case management interventions? And which contextual factors influence the success of case management interventions in individual primary care settings or EDs?

To mark this recent shift in the field, in 2019, Hudon and colleagues published a systematic review addressing the first of these questions: Which characteristics of case management interventions lead to positive outcomes [11]? What their analysis revealed was that case management interventions produced the most positive outcomes if they combined appropriate case finding (i.e. targeted the right individuals) with a high-intensity case management intervention and/or a multidisciplinary, inter-organizational care plan. This review has helped refine our understanding of which aspects of case management interventions contribute to success, clarifying that not all case management interventions are created equal, but further questions remain. For instance, case management is known to be a complex intervention with a multitude of components that interact to produce outcomes that are highly dependent on the context of individual care settings. But what are these contextual factors and which ones facilitate or hinder the implementation and success of case management? To address these emerging questions, research is well underway.

For instance, a systematic review conducted by a team of Canadian researchers exploring barriers and facilitators to the implementation of case management in primary care settings will soon be published providing a valuable contribution to the literature (Hacker Teper M, Vedel I, Yang $\mathrm{X}$, Margo-Dermer E, Hudon C. Understanding barriers and facilitators to case management in primary care: a systematic reivew and thematic synthesis. Annals of Family Medicine 2020; In Press). Further, two ongoing trials are helping advance the field. First, at the University of Lausanne, a multi-site trial called the I-CaM project (Implementing a Case Management Intervention for Frequent User of the Emergency Department) has been recruiting participants since 2018 [12]. The trial's objectives are twofold: (1) to develop and implement a case management intervention tailored to frequent users across multiple EDs, and (2) to evaluate the implementation process and effectiveness. Though final results will not be available until later this year, it has become clear that contextual factors significantly influence implementation at individual care settings. These findings, once published, will provide key insights into the influence of contextual factors, as well as implementation strategies, for case management interventions across diverse healthcare settings.

Second, with a focus on primary care-based case management interventions, Hudon and colleagues in Canada are leading a nation-wide multi-site study with three stated objectives: (1) to identify facilitators and barriers of implementation, (2) to explain and understand the relationship between actors, contextual factors, mechanisms and outcomes of the intervention, and (3) to identify next steps towards the spread of case management in primary care (13). These studies, just a few examples amongst many, mark an exciting shift in the field of case management aimed at reducing frequent $\mathrm{ED}$ use.

Although we have made significant progress in the field of frequent ED use since the Pines editorial, knowledge gaps remain including some which Pines and colleagues highlighted a decade ago. Ongoing gaps include,

1. A lack of sub-categorization of frequent ED use that would facilitate a more in-depth understanding of various epidemiologic patterns underlying heterogeneous frequent user populations. As suggested by Pines, these categories could include frequency and pattern of ED use, insurance status, and use of one or more EDs as a regular source of care.

2. Limited availability of qualitative data on topics such as root causes of frequent ED use from the perspective of frequent users, the impact of case management from the perspective of frequent users and healthcare providers, including satisfaction with the intervention and views regarding its long-term sustainability.

3. Incomplete knowledge of best approaches and ideal strategies for effective implementation of case management.

4. Limited understanding of other key topics including which healthcare professionals are best suited to serve as case managers (i.e. nurses, social workers, community health workers, etc.), optimal criteria for recruitment of frequent ED users to case management interventions, and advantages and disadvantages of implementing case management as primary care versus predominantly EDbased interventions.

Our reflections on where the field of frequent ED use is headed would not be complete without addressing the current global pandemic. Some might argue that faced with this international public health emergency interventions such as case management are a luxury we cannot afford and should be put on hold so that all healthcare efforts can go towards the provision of essential frontline care. This may be needed in these initial months; however, if we sideline such interventions for long, we run the risk of leaving behind society's most vulnerable patients, including frequent users of the 
ED, many of whom are being disproportionately impacted medically and economically by the pandemic. The previous challenges that frequent ED users faced in accessing and receiving high-quality care have quickly become even more formidable amidst the pandemic. Instead of side-lining case management and related multidisciplinary interventions aimed at supporting co-morbid and socially complex patients, we must ensure their continuity, quickly adapting them so they target those who are most vulnerable.

Overall, the field of frequent ED use has evolved considerably over the past 10 years. Based on increasingly robust evidence, it appears that we can now collectively shift from questioning whether or not case management is effective at reducing frequent ED use and related healthcare costs to asking questions about the where, when and how of case management. Studies underway, as well as future research that addresses this shift, will help us move towards broader implementation of this important intervention in primary care and ED settings around the world. Further, we should challenge ourselves to revisit the gaps that remain in the field of case management to ensure our most vulnerable patients can receive the care and support they need to achieve optimal health outcomes during these particularly challenging times and beyond.

\section{Compliance with ethical standards}

Conflict of interest The authors declare no conflict of interest.

\section{References}

1. Pines JM, Asplin BR, Kaji AH, Lowe RA, Magid DJ, Raven M, et al. Frequent users of emergency department services: gaps in knowledge and a proposed research agenda. Acad Emerg Med. 2011;18(6):e64-9.

2. Locker TE, Baston S, Mason SM, Nicholl J. Defining frequent use of an urban emergency department. Emerg Med J. 2007;24(6):398-401.
3. Althaus F, Paroz S, Hugli O, Ghali WA, Daeppen JB, PeytremannBridevaux I, et al. Effectiveness of interventions targeting frequent users of emergency departments: a systematic review. Ann Emerg Med. 2011;58(1):41-52 e42.

4. Kumar GS, Klein R. Effectiveness of case management strategies in reducing emergency department visits in frequent user patient populations: a systematic review. J Emerg Med. 2013;44(3):717-29.

5. Tricco AC, Antony J, Ivers NM, Ashoor HM, Khan PA, Blondal E, et al. Effectiveness of quality improvement strategies for coordination of care to reduce use of health care services: a systematic review and meta-analysis. CMAJ. 2014;186(15):E568-78.

6. Soril LJ, Leggett LE, Lorenzetti DL, Noseworthy TW, Clement FM. Reducing frequent visits to the emergency department: a systematic review of interventions. PLoS ONE. 2015;10(4):e0123660.

7. Raven MC, Kushel M, Ko MJ, Penko J, Bindman AB. The effectiveness of emergency department visit reduction programs: a systematic review. Ann Emerg Med. 2016;68(4):467-83 e15.

8. Van den Heede K, Van de Voorde C. Interventions to reduce emergency department utilisation: a review of reviews. Health Policy. 2016;120(12):1337-49.

9. Hudon C, Chouinard MC, Lambert M, Dufour I, Krieg C. Effectiveness of case management interventions for frequent users of healthcare services: a scoping review. BMJ Open. 2016;6(9):e012353.

10. Moe J, Kirkland SW, Rawe E, Ospina MB, Vandermeer B, Campbell $\mathrm{S}$, et al. Effectiveness of interventions to decrease emergency department visits by adult frequent users: a systematic review. Acad Emerg Med. 2017;24(1):40-52.

11. Hudon C, Chouinard MC, Pluye P, El Sherif R, Bush PL, Rihoux $\mathrm{B}$, et al. Characteristics of case management in primary care associated with positive outcomes for frequent users of health care: a systematic review. Ann Fam Med. 2019;17(5):448-58.

12. Grazioli VS, Moullin JC, Kasztura M, Canepa-Allen M, Hugli $\mathrm{O}$, Griffin J, et al. Implementing a case management intervention for frequent users of the emergency department (I-CaM): an effectiveness-implementation hybrid trial study protocol. BMC Health Serv Res. 2019;19(1):28.

13. Hudon C, Chouinard MC, Aubrey-Bassler K, Burge F, Doucet S, Ramsden VR, et al. Case management in primary care for frequent users of healthcare services with chronic diseases and complex care needs: an implementation and realist evaluation protocol. BMJ Open. 2018;8(11):e026433. 